\title{
Spontaneous Magnetization and Phase Diagrams of the Mixed Spin-1/2 and Spin-S Ising Model on the Bethe Lattice
}

\author{
J. STREČKA ${ }^{a}$ AND C. EKIZ ${ }^{b, *}$ \\ ${ }^{a}$ Department of Theoretical Physics and Astrophysics, Faculty of Science, P.J. Šafárik University, \\ Park Angelinum 9, 04001 Košice, Slovak Republic \\ ${ }^{b}$ Department of Physics, Faculty of Science and Art, Adnan Menderes University, 09010 Aydın, Turkey \\ The effect of uniaxial single-ion anisotropy on magnetic and critical properties of the mixed spin-1/2 and spin- \\ $S(S>1 / 2)$ Ising model on a three-coordinated Bethe lattice is rigorously examined with the help of star-triangle \\ transformation and exact recursion relations. In particular, our attention is focused on the ferrimagnetic version of \\ the model, which exhibits diverse temperature dependences of the total and both sublattice magnetizations. It is \\ shown that the critical behavior of the mixed-spin Ising model on the Bethe lattice basically depends on whether \\ the quantum spin number $S$ is integer or half-odd-integer.
}

DOI: 10.12693/APhysPolA.131.615

PACS/topics: $05.50 .+\mathrm{q}, 75.10 . \mathrm{Hk}, 75.10 . \mathrm{Kz}, 75.40 . \mathrm{Cx}$

\section{Introduction}

The mixed-spin Ising models traditionally attract a great deal of research interest, because they may exhibit much more complex magnetic and critical behavior than their single-spin counterparts [1]. Despite considerable efforts, there exist only a few fully exactly tractable mixed-spin Ising models defined mostly on three-coordinated lattices such as the honeycomb [2], bathroom-tile [3] and square-hexagon-dodecagon [1] lattice. In the present work, we will exactly solve the mixed spin-1/2 and spin- $S$ Ising model on a three-coordinated Bethe lattice with the aim to study an influence of the spin magnitude $S$ and single-ion anisotropy upon the critical behavior and temperature dependences of the spontaneous magnetization.

\section{Mixed-spin Ising model and its exact solution}

Let us consider the mixed spin- $1 / 2$ and spin- $S$ Ising model on the Bethe lattice with the coordination number $q=3$, which is schematically illustrated on the left-handside of Fig. 1. The investigated Bethe lattice consists of two inequivalent interpenetrating sublattices $\mathrm{A}$ and $\mathrm{B}$, which are occupied by the spin- $1 / 2$ and spin- $S$ atoms depicted in Fig. 1 as filled and empty circles, respectively. The total Hamiltonian of the mixed spin- $1 / 2$ and spin- $S$ Ising model on the three-coordinated Bethe lattice reads

$$
\mathcal{H}=J \sum_{\langle i j\rangle}^{3 N / 2} S_{i} \sigma_{j}-D \sum_{i=1}^{N} S_{i}^{2},
$$

where $N$ is the total number of lattice sites at each sublattice, $S_{i}=-S,-S+1, \ldots, S$ and $\sigma_{j}= \pm 1 / 2$ are the Ising spins located at the $i$-th and $j$-th lattice point of the sublattice A and B, respectively. The first summation is

\footnotetext{
*corresponding author; e-mail: cekiz@adu.edu.tr
}

carried out over all nearest-neighbor spin pairs coupled via the antiferromagnetic interaction $J>0$ and the second summation takes into account the uniaxial single-ion anisotropy $D$ acting on the spins from the sublattice B.

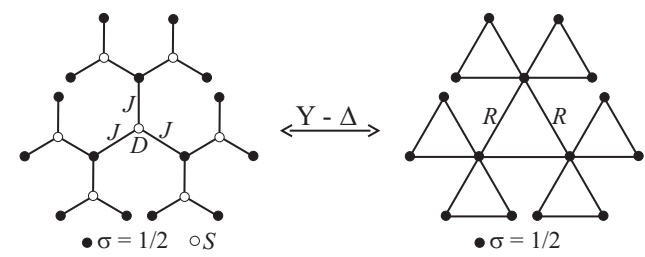

Fig. 1. The mixed spin-1/2 and spin- $S$ Ising model on the Bethe lattice is mapped via the star-triangle transformation on the spin- $1 / 2$ Ising model on the triangular Husimi lattice.

The total Hamiltonian (1) can be rewritten as a sum over the site Hamiltonians $\mathcal{H}=\sum_{i=1}^{N} \mathcal{H}_{i}$, where each site Hamiltonian $\mathcal{H}_{i}$ involves all the interaction terms connected to the $i$-th spin- $S$ atom from the sublattice B

$$
\mathcal{H}_{i}=J S_{i}\left(\sigma_{i 1}+\sigma_{i 2}+\sigma_{i 3}\right)-D S_{i}^{2} .
$$

The partition function of the mixed spin- $1 / 2$ and spin$S$ Ising model on the Bethe lattice can be subsequently partially factorized into the product

$$
\mathcal{Z}=\sum_{\{\sigma\}} \prod_{i=1}^{N} \sum_{S_{i}=-S}^{S} \exp \left(-\beta \mathcal{H}_{i}\right)=\sum_{\{\sigma\}} \prod_{i=1}^{N} \mathcal{Z}_{i}
$$

where $\beta=1 /\left(k_{\mathrm{B}} T\right), k_{\mathrm{B}}$ is the Boltzmann constant, $T$ is the absolute temperature, $\sum_{\{\sigma\}}$ denotes a summation over all possible configurations of the spin- $1 / 2$ atoms from the sublattice A and the second summation $\sum_{S_{i}}$ is carried out over spin states of the $i$ th spin- $S$ atom from the sublattice B. After performing the latter particular summation one obtains the explicit form of the site partition function $\mathcal{Z}_{i}$, which can be subsequently replaced through the star-triangle transformation (see Ref. [1]): 


$$
\begin{gathered}
\mathcal{Z}_{i}=\sum_{n=-S}^{S} \exp \left(\beta D n^{2}\right) \cosh \left(\beta J n\left(\sigma_{i 1}+\sigma_{i 2}+\sigma_{i 3}\right)\right)= \\
\quad \exp \left(\beta R\left(\sigma_{i 1} \sigma_{i 2}+\sigma_{i 2} \sigma_{i 3}+\sigma_{i 3} \sigma_{i 1}\right)\right) .
\end{gathered}
$$

The mapping parameters $A$ and $R$ can be directly obtained from a self-consistency condition [1] of the startriangle transformation (4), which requires

$$
A=\left(V_{1} V_{2}^{3}\right)^{\frac{1}{4}}, \quad \beta R=2 \ln \left(V_{1} / V_{2}\right),
$$

whereas the expressions $V_{1}$ and $V_{2}$ are defined as follows:

$$
\begin{aligned}
& V_{1}=\sum_{n=-S}^{S} \exp \left(\beta D n^{2}\right) \cosh (3 \beta J n / 2), \\
& V_{2}=\sum_{n=-S}^{S} \exp \left(\beta D n^{2}\right) \cosh (\beta J n / 2) .
\end{aligned}
$$

The substitution of the star-triangle transformation (4) into the partition function (3) establishes a rigorous mapping relationship between the partition function $\mathcal{Z}$ of the mixed-spin Ising model on the three-coordinated Bethe lattice and the partition function $\mathcal{Z}_{\text {Husimi }}$ of the corresponding spin- $1 / 2$ Ising model on the triangular Husimi lattice with the effective pair interaction $R$ (see Fig. 1):

$$
\mathcal{Z}(\beta, J, D)=A^{N} \mathcal{Z}_{\text {Husimi }}(\beta, R) .
$$

Following the approach elaborated in Ref. [4] one may extract exact results for the mixed-spin Ising model on the Bethe lattice from a rigorous mapping correspondence (7) with the spin- $1 / 2$ Ising model on the triangular Husimi lattice, which can be exactly treated by the method of exact recursion relations [5, 6]. From this point of view, our exact solution of the mixed-spin Ising model on the Bethe lattice is formally completed, since the exact results for the critical temperature and spontaneous magnetization of the spin- $1 / 2$ Ising model on the triangular Husimi lattice were already reported in Refs. $[4,6]$. For instance, the phase diagrams of the mixed-spin Ising model on the three-coordinated Bethe lattice can be obtained from a comparison of the effective interaction given by Eqs. (5),(6) with the critical value $\beta_{\mathrm{c}} R=\ln (7 / 3)$ [4], which provides the following critical condition:

$$
\begin{gathered}
3 \sum_{n=-S}^{S} \mathrm{e}^{\beta_{\mathrm{c}} D n^{2}} \cosh \left(\frac{3}{2} \beta_{\mathrm{c}} J n\right)= \\
7 \sum_{n=-S}^{S} \mathrm{e}^{\beta_{\mathrm{c}} D n^{2}} \cosh \left(\frac{1}{2} \beta_{\mathrm{c}} J n\right) .
\end{gathered}
$$

The similar procedure can be utilized for the exact calculation of both spontaneous sublattice magnetizations $m_{\mathrm{A}}=\left\langle\sigma_{j}\right\rangle$ and $m_{\mathrm{B}}=\left\langle S_{i}\right\rangle$, which can be computed from the spontaneous magnetization of the spin- $1 / 2$ Ising model on the triangular Husimi lattice [6].

\section{Results and discussion}

Let us proceed to a discussion of the most interesting results for the phase diagrams and spontaneous magnetization of the mixed spin- $1 / 2$ and spin- $S$ Ising model on the three-coordinated Bethe lattice. Figure 2 illus- trates typical dependences of the reduced critical temperature $k_{\mathrm{B}} T_{\mathrm{c}} / J$ on a relative strength of the uniaxial single-ion anisotropy $D / J$ for several values of the quantum spin number $S$. As one can see, the critical temperature monotonically decreases with decrease of the parameter $D / J$ for any quantum spin number $S$. However, the critical temperature tends to zero as $D / J \rightarrow-3 / 2$ for all integer spins $S$ (Fig. 2a), while it tends towards the finite value $k_{\mathrm{B}} T_{\mathrm{c}} / J=1 / \ln 9$ in the asymptotic limit $D / J \rightarrow-\infty$ for all half-odd-integer spins $S$ (Fig. 2b). This qualitative difference appears owing to a sufficiently strong negative single-ion anisotropy $D / J$, which forces all integer spins towards their non-magnetic spin state $S_{i}=0$ and all half-odd-integer spins towards their lowest magnetic spin state $S_{i}=1 / 2$.
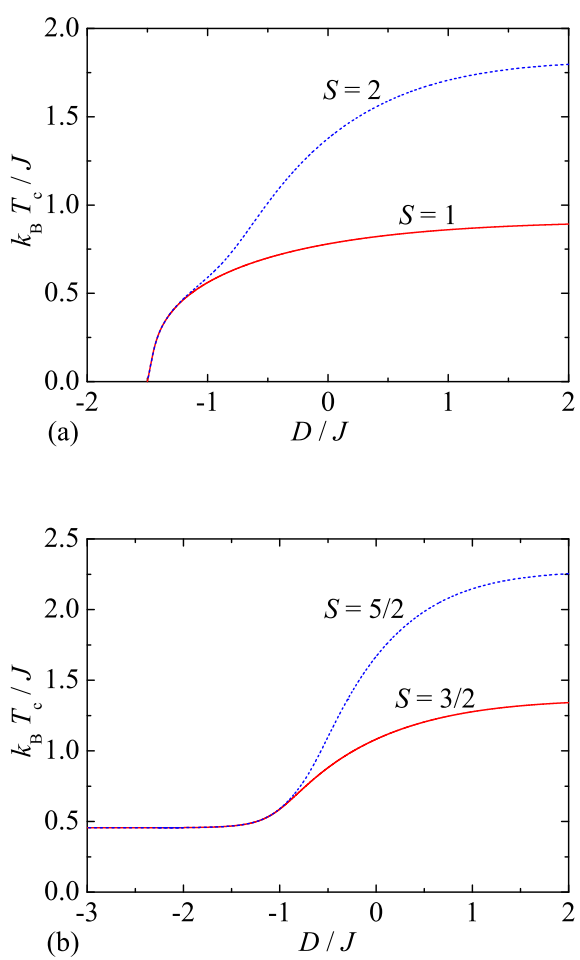

Fig. 2. The critical temperature as a function of the uniaxial single-ion anisotropy $D / J$ for a few different spin values: (a) $S=1$ and $S=2$, (b) $S=3 / 2$ and $S=5 / 2$.

Next, let us take a closer look at typical temperature variations of both sublattice magnetizations $\left(m_{\mathrm{A}}, m_{\mathrm{B}}\right)$ and total magnetization $m_{\mathrm{T}}=\left(m_{\mathrm{A}}+m_{\mathrm{B}}\right) / 2$, which can be classified according to the extended Néel nomenclature $[3,4]$. It can be deduced from Fig. 3 that a more rapid thermal decline of the sublattice magnetization $m_{B}$ caused by $D / J<0$ appears due to energetic favoring of the lower spin state(s). As a result, the usual Q-type thermal dependence of the total magnetization observable in Fig. 3a for the quantum spin number $S=1$ and non-negative values of the single-ion anisotropy (e.g. $D / J=1.0$ and 0.0 ) may change to more striking R- and S-type thermal dependences illustrated for $D / J=-1.0$ 

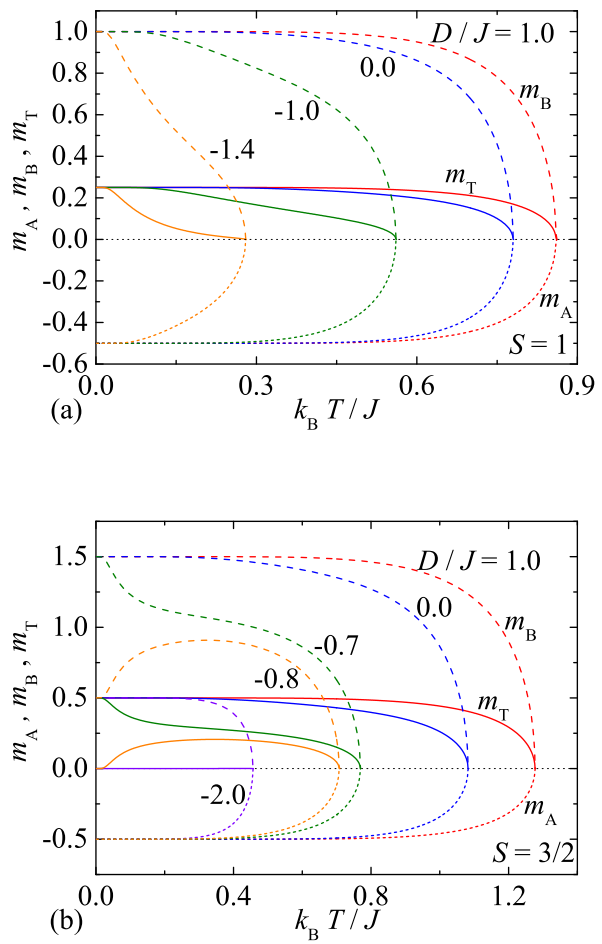

Fig. 3. Temperature variations of the total magnetization $m_{\mathrm{T}}$ (solid lines), the sublattice magnetization $m_{\mathrm{A}}$ (dotted lines) and $m_{\mathrm{B}}$ (dashed lines) for several values of the uniaxial single-ion anisotropy $D / J$ and two different values of the quantum spin number: (a) $S=1$, (b) $S=3 / 2$.

and -1.4 , respectively. Temperature variations of the spontaneous magnetization for another particular spin case $S=3 / 2$ depicted in Fig. 3b serve in evidence that the total magnetization may additionally display the peculiar L-type dependence. Under this circumstance, the total magnetization rises from zero due to thermal excitations from the lowest-energy spin state $S_{i}=1 / 2$ towards the higher-energy state $S_{i}=3 / 2$, whereas this effect is especially visible when $D / J \lesssim-0.75$ (see in Fig. 3b the curve for $D / J=-0.8)$. Note that the similar P-type dependences with thermally-induced increase of the total magnetization starting from the non-zero asymptotic value can be found for the spin magnitudes $S>3 / 2$.

\section{Conclusion}

The present work provides exact results for the phase diagrams, total and sublattice magnetizations of the ferrimagnetic version of the mixed spin- $1 / 2$ and spin- $S$ Ising model on the three-coordinated Bethe lattice. In particular, we have focused our attention to the effect of uniaxial single-ion anisotropy upon temperature dependences of the spontaneous magnetization and finite-temperature phase diagrams, which were established for several spin magnitudes $S$. It has been shown that the critical temperature exhibits a monotonous decline with decrease of the single-ion anisotropy $D / J$, whereas the overall critical behavior basically depends on whether the quantum spin number $S$ is integer or half-odd-integer. Besides, we have also proved an extraordinary diversity in thermal dependences of the total magnetization, which were classified as Q-, R-, P-, L- or S-type thermal dependences within the extended Néel nomenclature [3, 4]. By contrast, the $\mathrm{N}$ - and $\mathrm{W}$-type thermal dependences of the total magnetization with one or two compensation temperatures cannot be found within the investigated ferrimagnetic model. The present results for the mixed spin- $1 / 2$ and spin- $S$ Ising model on the three-coordinated Bethe lattice are in a good qualitative accord with the exact results obtained previously for the same model on the honeycomb [2] and bathroom-tile [3] lattices.

\section{Acknowledgments}

This work was financially supported under the grant VEGA 1/0331/15 and APVV-0097-12.

\section{References}

[1] J. Strečka, M. Jaščur, Acta Phys. Slov. 65, 235 (2015).

[2] L.L. Gonçalves, Phys. Scr. 32, 248 (1985).

[3] J. Strečka, Physica A 360, 379 (2006).

[4] C. Ekiz, J. Strečka, M. Jaščur, Cent. Eur. J. Phys. 7, 509 (2009).

[5] R.J. Baxter, Exactly Solved Models in Statistical Mechanics, Academic Press, New York 1982.

[6] E. Jurčišinová, M. Jurčišin, J. Stat. Phys. 147, 1077 (2012). 